\title{
The Kaon identification system at the NA62 CERN experiment
}

\author{
Emilie Maurice ${ }^{* \dagger}$ \\ University of Liverpool, \\ E-mail: emaurice@hep.ph.liv.ac.uk
}

\begin{abstract}
The main goal of the NA62 experiment at the CERN SPS accelerator is to measure the branching ratio of the ultra-rare $\mathrm{K}^{+} \rightarrow \pi^{+} v \bar{v}$ decay with $10 \%$ accuracy. This will be achieved by detecting about $100 \mathrm{~K}^{+} \rightarrow \pi^{+} v \bar{v}$ decays with a ratio of signal/background $\sim 10$ in 2 - 3 years of data taking. NA62 will use a $750 \mathrm{MHz}$ high-energy unseparated charged hadron beam, with a $6 \%$ kaons component, and a kaon decay-in-flight technique. The kaons must be identified, together with a precise timestamp, in order to guarantee the rejection of background induced by accidental overlap of events in the detector. This is achieved by the kaon identification system, KTAG, which identifies kaons with an efficiency higher than $95 \%$ and precise time information with a resolution better than 100 ps. KTAG re-uses the Cherenkov radiator and optics of a CEDAR, a ring-focusing Cherenkov detector designed for MHz beam intensity in the 1970s. To reach the required performance, KTAG is equipped with new photon detectors, electronics readout, mechanics, cooling and safety systems. The performance of KTAG is presented after setting the detector within the context of the NA62 experiment.
\end{abstract}

The European Physical Society Conference on High Energy Physics

22-29 July 2015

Vienna, Austria

* Speaker.

${ }^{\dagger}$ On behalf the NA62 Collaboration 


\section{Kaon Physics at NA62}

Kaon physics has played a key role in the development of the Standard Model. High-precision studies of rare kaon decays are sensitive to new physics processes in a complementary way to the direct searches of LHC. The NA62 experiment, based at CERN SPS accelerator has been designed to collect a very large sample of $\mathrm{K}^{+}$decays with a sensitivity to branching ratio smaller than $10^{-10}$. In particular, NA62 will measure the branching ratio of the decay $\mathrm{K}^{+} \rightarrow \pi^{+} v \bar{v}$. This ultra rare decay is a flavour-changing neutral current process forbidden at tree level. Its branching ratio is predicted precisely by the Standard Model : $B R_{S M}=(9.11 \pm 0.72) \times 10^{-11}$ [1]. A significant deviation of the measured value will provide evidence of physics beyond the Standard Model. The latest measurement of this branching ratio was made by the collaborations E787 and E949, using a stopped-kaon technique, with 7 observed $\mathrm{K}^{+} \rightarrow \pi^{+} v \bar{v}$ events : $B R=\left(17.3_{-10.5}^{+11.5}\right) \times 10^{-11}$ [2]. NA62 aims to collect more than 100 signal events in $2-3$ years of data taking, and will measure this branching ratio with $10 \%$ accuracy.

\section{The NA62 CERN Experiment}

The NA62 experiment is located at CERN in the North area and uses a primary proton beam from the SPS for the production of a secondary kaon beam. The beam and the detector are set up to optimize the study of the $\mathrm{K}^{+} \rightarrow \pi^{+} v \bar{v}$ decays. NA62 uses the decay-in-flight technique with high momentum kaons $(75 \mathrm{GeV} / \mathrm{c})$. This choice was driven by the improved background rejection possible with high-energy decay products. To reach a sensitivity to branching ratio of $\mathrm{O}\left(10^{-10}\right)$, the rate of kaons decaying must be $\sim 5 \mathrm{MHz}$. As only $10 \%$ of the kaons decay in the $65 \mathrm{~m}$ fiducial region between the upstream and downstream detectors, a $50 \mathrm{MHz}$ beam of $\mathrm{K}^{+}$is required. It is obtained with the secondary beam from the SPS which has a rate of $750 \mathrm{MHz}$, and consists of $6 \%$ $\mathrm{K}^{+}, 34 \% p, 60 \% \pi^{+}[3]$. Therefore it is essential to have an accurate timing (around $100 \mathrm{ps}$ ) of the kaon to precisely match the particles from the decay, and to avoid wrong association at a level below $1 \%$. To identify the $\mathrm{K}^{+} \rightarrow \pi^{+} v \bar{v}$ signal events and suppress the background, NA62 uses techniques based on kinematics and particle identification. To reduce the background due to neutral pions and muons in the final state, hermetic coverage enables vetoing of photons and muons.

The layout of the NA62 detector is presented in Figure 1. Three stages can be identified:

- first, a set of subdetectors dedicated to the beam kaon measurement;

- second, a fiducial region in vacuum housing the reconstructed kaon decay vertex;

- third, a spectrometer and particle identification detectors focusing on the decay products.

All these components are surrounded by photon detectors that can be used to veto unwanted decay modes.

The identification of $\mathrm{K}^{+} \rightarrow \pi^{+} v \bar{v}$ signal events requires a precise measurement of the beam properties before the fiducial region and the reconstruction of the $\pi^{+}$after the kaon decay. The beam particles are identified by the KTAG described in the next section. The Gigatracker, identified on Figure 1 by GTK, is composed of 3 stations of thin silicon-pixel detectors. It measures the momentum of the kaon with a $0.4 \%$ resolution, its flight direction with $16 \mu$ rad resolution, 
and its time with a resolution of 200 ps. The Chanti detector is a guard-ring scintillating counter surrounding the last GTK station to veto beam particles, which scatter inelastically in the material of the GTK stations. Its expected efficiency in vetoing signal-like events is $99 \%$. After the fiducial region, the momentum and coordinates of the secondary charged particles are measured with high accuracy by 2 pairs of 2 straw chambers, see Figure 1, separated by a dipole magnet which provides a vertical B-field of $0.4 \mathrm{~T}$. The expected momentum resolution of this spectrometer is $\sigma(p) / p \sim 0.02 \%$

Because of the low-mass tracking chambers, kinematics enables a reduction in background by a factor of $10^{4}$, and minimizes the inelastic scattering of beam particle, which can mimic a signal event. The muon veto system is crucial both at the trigger level and offline to remove $\mathrm{K}^{+} \rightarrow \mu^{+} v\left(\pi^{0}\right)$. It consists of a plane of fast scintillators (MUV3), and is complemented by two hadron calorimeters (MUV1 and MUV2) which are designed to measure the energy deposited and help to distinguish hadron showers from muons. The identification of electromagnetic showers and the precise measurement of their energy are ensured by the NA48 liquid krypton calorimeter, $\mathrm{LKr}$ in Figure 1, with new readout electronics. The vetoing of photons with an angle higher than $8 \mathrm{mrad}$ with respect to the beam is done by 12 rings of lead glass counters surrounding the decay region. It requires an inefficiency no higher than $10^{-4}$ for high energy photons. Photons with very small angle which are converted in the upstream material are vetoed downstream of MUV3 by the Intermediate Ring Calorimeter (IRC) and the Small Angle Calorimeter (SAC). Both are Shashlyk-type calorimeters. An additional reduction factor of $10^{2}$ comes from the RICH counter which is a ring imaging Cherenkov, used to separate pions from muons between 15 and $35 \mathrm{GeV} / \mathrm{c}$. It consists of a $17 \mathrm{~m}$ long radiator filled with Neon Gas at $1 \mathrm{~atm}$. A high-performance trigger and a data-acquisition system complete the NA62 detector.

In 2012, a technical run was carried out to study the performances of NA62 subdetectors and finalize their design, in particular these of KTAG [4]. A pilot run took place in autumn 2014 to commission the full detector and the readout system. KTAG was fully commissioned and its performance found to meet the design requirements. In June 2015, NA62 began to take physics data, and this will continue through 2018.

\section{The Kaon identification system}

The Kaon identification system (KTAG), see Figure 2 (left), suppresses the accidental nonkaon background and defines the offline timestamp for all subdetectors with a precision better than 100 ps. KTAG identifies the $50 \mathrm{MHz}$ of $\mathrm{K}^{+}$within an unseparated beam of $750 \mathrm{MHz}$. For each $\mathrm{K}^{+}$, there are on average 20 detected photons to meet the design requirement, which are : $\mathrm{K}^{+}$ identification with an efficiency higher than $95 \%$, kaon time resolution better than $100 \mathrm{ps}$, and a particle misidentification lower than $10^{-4}$.

The KTAG is based on a ring-focusing Cherenkov counter (CEDAR for Cherenkov Detector with Achromatic Ring focus [5]) equipped with new photon detectors and read-out system. This counter is optimized to detect Cherenkov light from $\mathrm{K}^{+}$with an internal optical system focusing the light on the photon detector plane. The pressure of the radiator gas is then used to distinguish the particles of different velocities so to identify $\mathrm{K}^{+}$. 


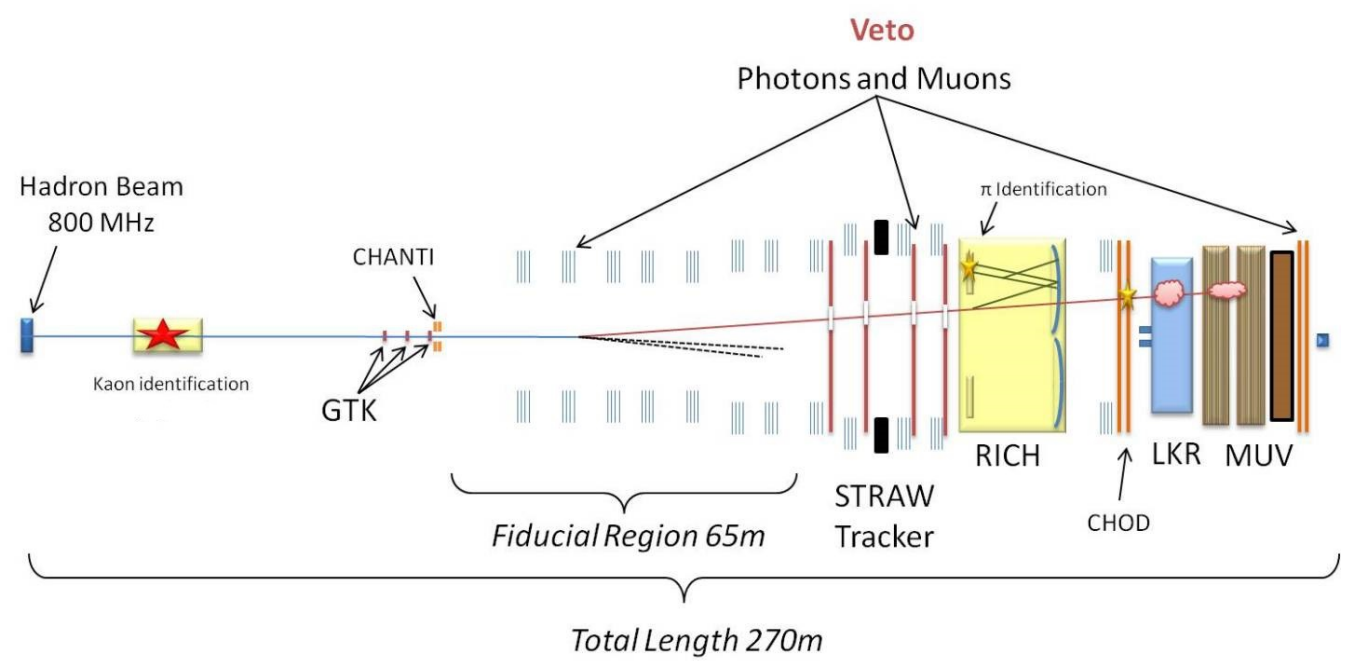

Figure 1: Schematic view of the NA62 experiment with the main sub-detectors.

As shown on the Figure 2 (right), the Cherenkov light produced in the gas is reflected by a mirror, then passes through a corrector lens and finally through a diaphragm. The lens corrects for the optical dispersion in the $\mathrm{N}_{2}$ gas and ensures that the Cherenkov light, emitted at all wavelengths, arrives at the same radius on the diaphragm plane. The aperture of the diaphragm is fixed in such a way that particle of different masses are selected by varying the gas pressure. In consequence, light from unwanted particles hits the diaphragm at different radii from $\mathrm{K}^{+}$and does not pass through the diaphragm; hence only the wanted $\mathrm{K}^{+}$produce light to illuminate the PMTs. The pressure scan highlighting the $\mathrm{K}^{+}$selection is shown on Figure 3 (left) : the optimal $\mathrm{N}_{2}$ gas pressure is $1.74 \mathrm{bar}$ to select $75 \mathrm{GeV} / \mathrm{c} \mathrm{K}^{+}$. After the diaphragm, the light passes through additional lenses and light guides to finally reach eight light-boxes where the photon detectors (PMT) are located, see details on Figure 3 (right). They are situated in an insulated, cooled Faraday enclosure flushed with $\mathrm{N}_{2}$. Each box contains 32 R9880 Hamamatsu PMTs and 16 R7400 Hamamatsu PMTs. The rate of photons on each PMT is then reduced to $5 \mathrm{MHz}$, which can be read out and digitized. Thus KTAG is able to handle the high beam flux.
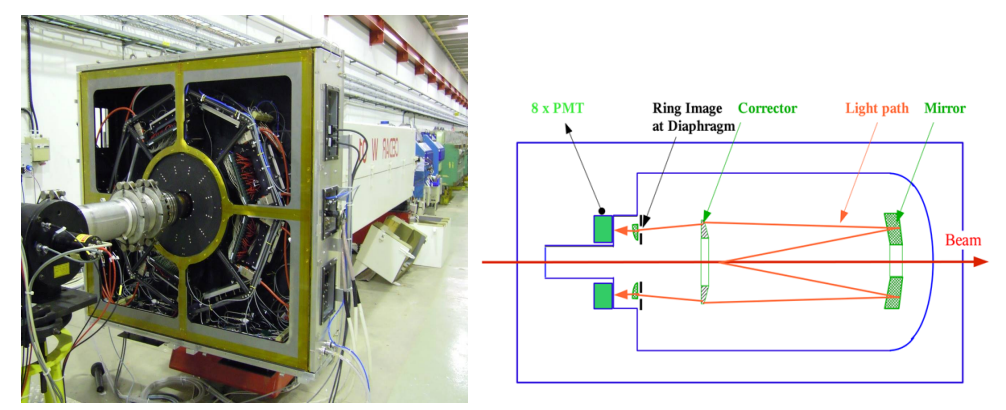

Figure 2: KTAG in the NA62 setup (left) and a schematic view of the light path in the CEDAR (right).

The data from the pilot run have been analyzed to assess the performance of KTAG. This 

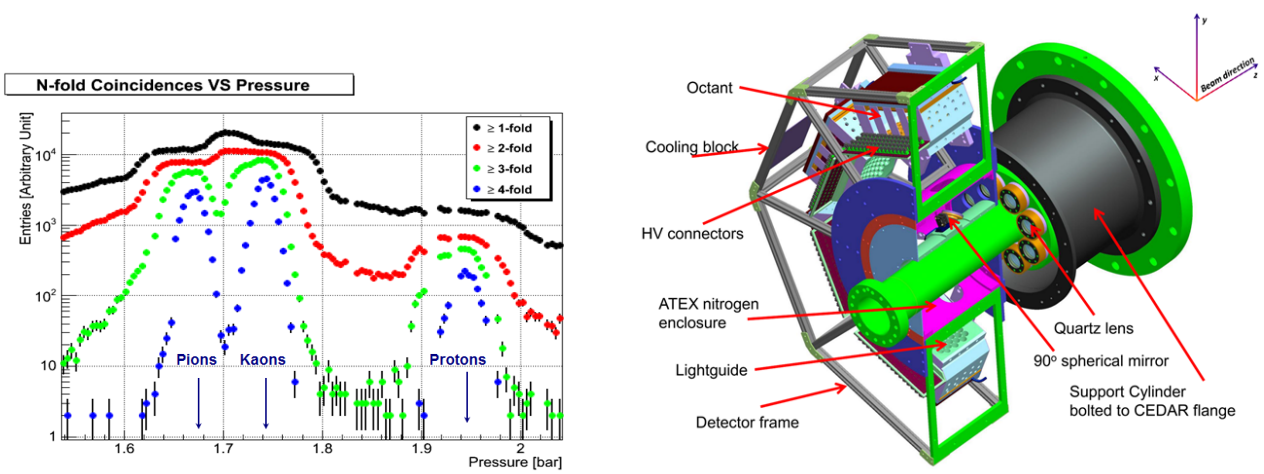

Figure 3: Result of the scan of the gas pressure highlighting the separation between $\mathrm{K}^{+}, \pi^{+}, p$ in $\mathrm{N}_{2}$ (left), and layout of the KTAG (right).

evaluation uses the $\mathrm{K}^{+} \rightarrow \pi^{+} \pi^{0}$ decays reconstructed using only the $\mathrm{LKr}$ calorimeter and assuming the nominal beam parameters. Time resolution of the signal from a PMT is $280 \mathrm{ps,} \mathrm{see} \mathrm{Figure} 4$ (left), leading to a time resolution of about 70 ps when collecting 20 photons to detect a $\mathrm{K}^{+}$, significantly better than the design expectations. The $\mathrm{K}^{+}$is identified using 5 (of the 8 ) coincident sectors in order to remove background from $\pi^{+}$, and as shown in Figure 4 (right), the efficiency is approximately $99 \%$. Data analysis has shown that $\pi^{+}$misidentification is lower than $10^{-4}$.
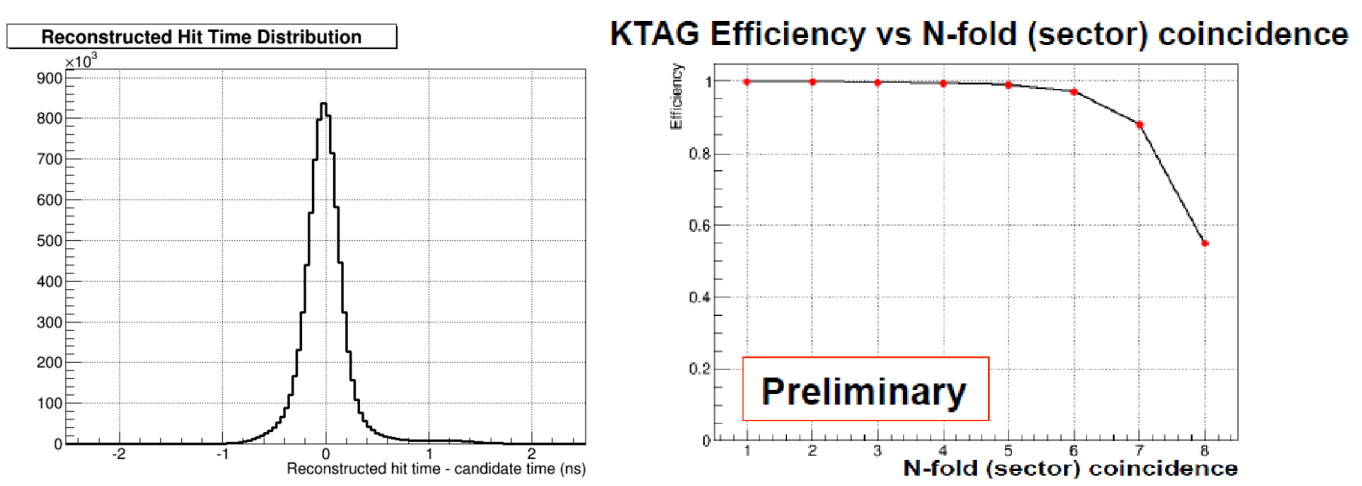

Figure 4: Preliminary analysis of the pilot run to assess KTAG performance : time resolution (left), $\mathrm{K}^{+}$ efficiency as a function of the sectors coincidences (right).

\section{Preliminary analysis with KTAG}

During the pilot run, almost all sub-detectors were installed and commissioned using a low intensity beam. These data were useful to test and calibrate the subsystems. KTAG was fully commissioned and its performance met the design expectations. A single track analysis was performed using only the spectrometer and KTAG information, assuming the nominal beam parameters [6]. Figure 5 shows the distribution of the angle between the kaon and the secondary track as a function of the track momentum. On the right side where a kaon is identified by KTAG, we clearly see the 3 kaon decays : $\mathrm{K}^{+} \rightarrow \pi^{+} \pi^{0}, \mathrm{~K}^{+} \rightarrow \mu^{+} v$ and $\mathrm{K}^{+} \rightarrow \pi^{+} \pi^{+} \pi^{-}$, whereas on the left side where kaon is 
anti-selected, we see only the accidental background and the scattered beam particles. From these figures, we can compute the ratio between the kaon beam component at $75 \mathrm{GeV} / \mathrm{c}$ and the pion beam component from the anti-selected events. This ratio is approximately 0.1 , in agreement with the measured composition of the beam. This preliminary analysis illustrates that KTAG performs well in selecting genuine $\mathrm{K}^{+}$decays.
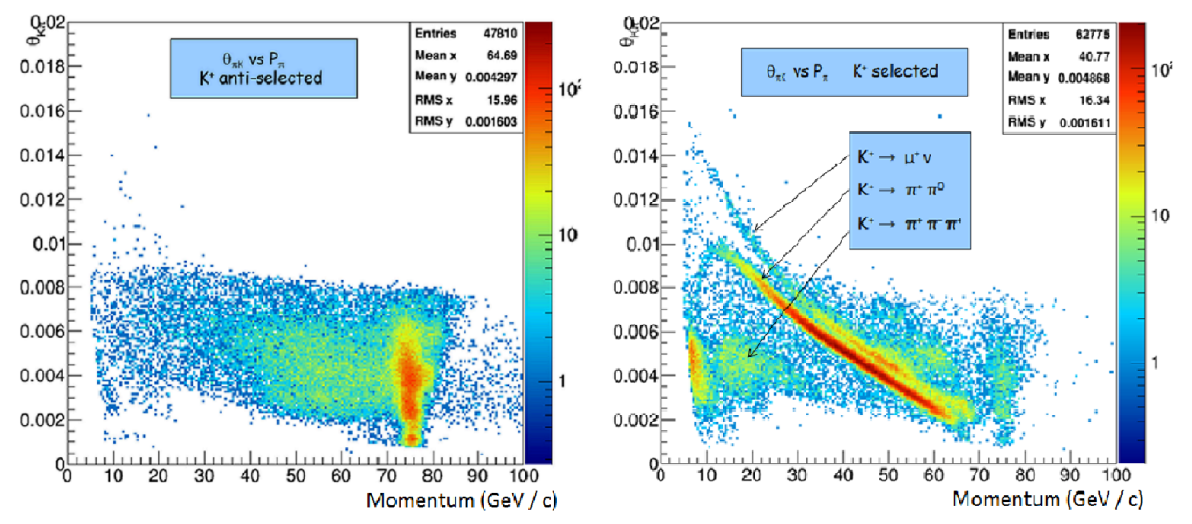

Figure 5: Single track analysis of the pilot run. On left : the anti-selected KTAG candidates, on the right the selected KTAG candidates.

\section{Conclusion}

The NA62 experiment has been optimized for the decay $\mathrm{K}^{+} \rightarrow \pi^{+} v \bar{v}$. To reach the sensitivity to branching ratio of $\mathrm{O}\left(10^{-10}\right)$, precise timing of the kaon and suppression of the accidental background are essential. These requirements are significantly fulfilled by the KTAG detector, which is a further development of a CEDAR counter operating in a high rate environment. A preliminary evaluation of its performance using the 2014 pilot run demonstrates that the kaon time resolution is approximately $70 \mathrm{ps}$, the identification efficiency is around $99 \%$, and the $\pi^{+}$misidentification less than $10^{-4}$, all of which are within the design expectations.

\section{References}

[1] A. J. Buras, D. Buttazzo, J. Girrbach-Noe, R. Knegjens, $\mathrm{K}^{+} \rightarrow \pi^{+} v \bar{v}$ and $\mathrm{K}_{L}^{0} \rightarrow \pi^{0} v \overline{n u}$ in the Standard Model: Status and Perspectives, arXiv:1503.02693

[2] E949 Collaboration, PRL101 (2008) 191802, arXiv:0808.2459

[3] Hahn F. et al. (NA62 Collaboration) 2010 NA62: Technical Design Document, NA62-10-07 https://cds.cern.ch/record/1404985

[4] Goudzovski E. et al. (NA62 Collaboration), Development of the kaon tagging system for the NA62 experiment at CERN, Nucl. Instrum. Meth. A801 (2015) 86, arXiv:1509.03773

[5] Bovet et al. 1982 The CEDAR counters for particle identification in the SPS secondary beams: a description and an operation manual, CERN-1982-013

[6] NA62 Collaboration, 2015 NA62 Status report to the CERN SPSC, CERN-SPSC-2015-012 (SPSC-SR-157) 